\title{
A novel functional site of extracellular matrix metalloproteinase inducer (EMMPRIN) that limits the migration of human uterine cervical carcinoma cells
}

\author{
TAKASHI SATO ${ }^{1}$, MAMI WATANABE ${ }^{1}$, KEI HASHIMOTO ${ }^{1}$, TOMOKO OTA ${ }^{1}$, NORIKO AKIMOTO ${ }^{1}$, \\ KEISUKE IMADA ${ }^{1}$, MOTOYOSHI NOMIZU ${ }^{2}$ and AKIRA ITO ${ }^{1}$ \\ ${ }^{1}$ Department of Biochemistry and Molecular Biology, ${ }^{2}$ Laboratory of Clinical Biochemistry, \\ School of Pharmacy, Tokyo University of Pharmacy and Life Sciences, Tokyo, Japan
}

Received July 1, 2011; Accepted August 12, 2011

DOI: 10.3892/ijo.2011.1224

\begin{abstract}
EMMPRIN (extracellular matrix metalloproteinase inducer)/CD147, a membrane-bound glycoprotein with two extracellular loop domains (termed loops I and II), progresses tumor invasion and metastasis by increasing the production of matrix metalloproteinase (MMP) in peritumoral stoma cells. EMMPRIN has also been associated with the control of migration activity in some tumor cells, but little is known about how EMMPRIN regulates tumor cell migration. In the present study, EMMPRIN siRNA suppressed the gene expression and production of EMMPRIN in human uterine cervical carcinoma SKG-II cells. An in vitro scratch wound assay showed enhancement of migration of EMMPRIN-knockdown SKG-II cells. In addition, the SKG-II cell migration was augmented by adding an E. coli-expressed human EMMPRIN mutant with two extracellular loop domains (eEMP-I/II), which bound to the cell surface of SKG-II cells. However, eEMP-I/II suppressed the native EMMPRIN-mediated augmentation of proMMP-1/procollagenase-1 production in a co-culture of the SKG-II cells and human uterine cervical fibroblasts, indicating that the augmentation of SKG-II cell migration resulted from the interference of native EMMPRIN functions by eEMP-I/ II on the cell surface. Furthermore, a systematic peptide screening method using nine synthetic EMMPRIN peptides coding the loop I and II domains (termed EM1-9) revealed that EM9 $\left({ }^{170}\right.$ HIENLNMEADPGQYR $\left.{ }^{184}\right)$ facilitated SKG-II cell migration. Moreover, SKG-II cell migration was enhanced by administration of an antibody against EM9, but not EM1 which is a crucial site for the MMP inducible activity of EMMPRIN. Therefore, these results provide novel evidence that EMMPRIN
\end{abstract}

Correspondence to: Dr Takashi Sato, Department of Biochemistry and Molecular Biology, School of Pharmacy, Tokyo University of Pharmacy and Life Sciences, 1432-1 Horinouchi, Hachioji, Tokyo 192-0392, Japan

E-mail: satotak@toyaku.ac.jp

Key words: extracellular matrix metalloproteinase inducer, tumor cell migration, human uterine cervical carcinoma cells, siRNA, systematic peptide screening method on the cell surface limits the cell migration of human uterine cervical carcinoma cells through ${ }^{170} \mathrm{HIENLNMEADPGQYR}{ }^{184}$ in the loop II domain. Finally, these results should provide an increased understanding of the functions of EMMPRIN in malignant cervical carcinoma cells, and could contribute to the development of clinical strategies for cervical cancer therapy.

\section{Introduction}

Extracellular matrix metalloproteinase inducer (EMMPRIN) (also known as CD147 or basigin) is a membrane-bound glycoprotein with two extracellular loop domains (termed loops I and II), which belongs to the immunoglobulin superfamily $(1,2)$. EMMPRIN is expressed on the cell surface of various tumors and some normal tissues/cells such as uterine endometrium, macrophages, and T-lymphocytes (1,3-5). Many investigators have reported that increased EMMPRIN expression causes the augmentation of matrix metalloproteinases (MMPs)-1, -2 , and -3 , and membrane-type MMP (MT-MMP) expression in peritumoral stoma cells, and thereby increases tumor invasiveness in vivo and in vitro (6-9). Therefore, EMMPRIN has been suggested to be a candidate as a diagnostic molecule for the identification of the early dissemination and malignancy of tumor cells $(1,2,10)$.

The extracellular loop domains of EMMPRIN have been reported to participate in the control of its biological activities; e.g., invasion, cell adhesion, migration, and angiogenesis in various cell species (1). For instance, we have recently reported that ${ }^{42}$ SLNDSATEVTGHRWLK ${ }^{57}$ (termed EM1) in the loop I domain of EMMPRIN is an active site for the augmentation of MMP production by EMMPRIN (11). Koga et al (12) have also reported similar findings, in that a synthetic peptide with 20 amino acids, including an EM1 sequence, abolishes the EMMPRINmediated proMMP-2 production. On the other hand, the loop II domain of EMMPRIN has been reported to form a complex with caveolin-1, which may be associated with the regulation of MMP production and the localization of EMMPRIN in lipid rafts (13-15). In addition, cyclophilin B-mediated isomerization of proline in the loop II domain of EMMPRIN has been reported to participate in the chemotactic activity of $\mathrm{T}$ lymphocytes (16). Although the identification of the functional site(s)/region(s) of 
EMMPRIN may help with the understanding of its pathophysiological roles and the development of anti-metastatic strategies, they remain to be elucidated.

Tumor cell migration is likely to be a crucial event for the progression of tumor invasion and metastasis $(17,18)$. Recent reports have also revealed that the augmented expression of EMMPRIN directly or indirectly stimulates tumor cell migration in vitro $(19,20)$. In addition, the interaction between EMMPRIN and membrane-associated molecules such as caveolin and annexin II on the cell surface has been reported to facilitate human carcinoma cell migration $(21,22)$. However, it is not well understood which extracellular domains of EMMPRIN are requisite for the control of tumor cell migration.

Since cervical cancer is the second most common cancer in women worldwide (23), the expression of EMMPRIN has been reported to correlate with the malignancy of cervical cancer $(11,24)$ and to be a marker for the evaluation of the recurrence and poor prognosis in cervical cancer patients (25). In the present study, we examined the regulation of tumor cell migration by EMMPRIN expressed on the cell surface of human cervical carcinoma SKG-II cells by carrying out gene silencing, competitive, and antibody-neutralizing experiments using: i) EMMPRIN siRNA, ii) E. coli-expressed human EMMPRIN mutant with two extracellular loop domains, iii) synthetic peptides coding extracellular domains of EMMPRIN, and iv) EMMPRIN antibodies. We found novel evidence that native EMMPRIN on the cell surface limits the cell migration of SKG-II cells through a functional site in the loop II domain.

\section{Materials and methods}

Cell culture and EMMPRIN siRNA treatment. Human uterine cervical carcinoma SKG-II cells (11) were cultured in Dulbecco's modified Eagle's medium (DMEM) (Invitrogen, Carlsbad, CA) supplemented with $10 \%$ fetal bovine serum (Thermo Electron, Waltham, MA), $200 \mathrm{U} / \mathrm{ml}$ of penicillin, and $200 \mu \mathrm{g} / \mathrm{ml}$ of streptomycin. To suppress EMMPRIN gene expression, SKG-II cells ( $3 \times 10^{5}$ cells in $60-\mathrm{mm}$ dishes) were transfected for $24 \mathrm{~h}$ with stealth EMMPRIN siRNA $(5 \mathrm{nM})$ or negative control siRNA (5 nM) (Invitrogen, cat. no. 10620312 and 12935112, respectively) after mixing with Hiperfect Transfection Reagent (Qiagen KK, Tokyo, Japan) according to the manufacturer's instructions. The siRNA transfected cells were subjected to real-time polymerase chain reaction (PCR), Western blot, and cell migration analyses.

Real-time PCR. Total RNA $(0.5 \mu \mathrm{g})$ was subjected to reverse transcriptase reaction using a PrimeScript RT reagent Kit (Takara Bio, Shiga, Japan) according to the manufacturer's instructions. Aliquots (an equivalent of $2.5 \mathrm{ng}$ of total RNA) of the transcript were subjected to real-time PCR using SYBR Premix Ex Taq II (Takara Bio) and QuantiTect Primer Assays [cat. no. QT00074564 for human EMMPRIN, and cat. no. QT00079247 for human glyceraldehydes-3-phosphate dehydrogenase (GAPDH), Qiagen KK]. The amplification cycle was performed at $94^{\circ} \mathrm{C}$ for $5 \mathrm{sec}$ and $60^{\circ} \mathrm{C}$ for $30 \mathrm{sec}$ using a Thermal Cycler Dice Real Time System TP-800 (Takara Bio). The obtained threshold cycle (CT) value was normalized by that of the GAPDH, and relative expression level was expressed as the mean value of control as $100 \%$.
Construct of E. coli-expressed EMMPRIN mutant with two extracellular loop domains. Human EMMPRIN mutant with two extracellular loop domains (EMP-I/II) was amplified by reverse transcriptase-PCR using previously cloned full-length EMMPRINcDNA(11) and the following specific primers: a sense primer, 5'-ATAGGAATCATGGCGGCTGCG-3' (6-26 bp) and an antisense primer, 5'-GTGGCTGCACACGCGGAGCGT-3' (629-609 bp). The amplified cDNA was inserted into a pBAD/ TOPO expression vector (pBAT-EMP-I/II), which included V5 and polyhistidine tags, using a pBAD TOPO ${ }^{\circledR}$ TA Expression kit (Invitrogen) according to the manufacturer's instructions. The pBAT-EMP-I/II plasmid was introduced into the E. coli, TOP10 competent cells (Invitrogen), and then the cells were cultured in the presence of $0.02 \%$ arabinose (Invitrogen) at $37^{\circ} \mathrm{C}$ for $6 \mathrm{~h}$. After centrifugation at $8,000 \mathrm{rpm}$ for $10 \mathrm{~min}$, the precipitated cells were disrupted in a lysis buffer $(2 \mathrm{mM}$ phosphate/10 $\mathrm{mM}$ imidazole/1\% Triton X-100) by sonication. After centrifugation at $10,000 \mathrm{rpm}$ for $10 \mathrm{~min}, E$. coli-expressing recombinant human EMP-I/II (eEMP-I/II) in the supernatant was purified using the ProBond $^{\mathrm{TM}}$ Purification System (Invitrogen) according to the manufacturer's instructions. Finally, the protein was dialyzed against $10 \%$ glycerol $/ \mathrm{Ca}^{2+}$ - and $\mathrm{Mg}^{2+}$-free phosphate-buffered saline $[\mathrm{PBS}(-)]$ and concentrated by ultrafiltration (cut-off $10 \mathrm{kDa})$.

Tumor cell migration assay. An in vitro scratch wound assay was performed to elucidate tumor cell migration. Briefly, SKG-II cells or siRNA-treated SKG-II cells ( $1 \times 10^{5}$ cells) in a 35-mm dish were cultured in DMEM supplemented with $10 \%$ fetal bovine serum, $200 \mathrm{U} / \mathrm{ml}$ of penicillin, and $200 \mu \mathrm{g} / \mathrm{ml}$ of streptomycin until grown to confluence. After washing the cells with DMEM, monolayers were scratched with a plastic pipette tip creating a cell-free area (wound) $\sim 2 \mathrm{~mm}$ in width. The cultures were washed twice in the same medium to remove cell debris and a marked area of the wound was photographed under phase-contrast microscopy prior to treatment. The cells were treated for $14 \mathrm{~h}$ with or without eEMP-I/II $(1$ and $2 \mu \mathrm{g} / \mathrm{ml})$, nine synthetic EMMPRIN peptides (termed EM1-EM9) $(60 \mu \mathrm{g} / \mathrm{ml})$ (Table I) (11), and antibodies against EM1 and EM9, which were customized by Operon Biotechnologies (Tokyo, Japan). After the treatments, the marked area of the wound was re-photographed and the migration distance was determined using an image analysis system, Lumina Vision (Ver. 2.2.2; Mitani, Fukui, Japan) according to the manufacturer's instructions.

Immunostaining for eEMP-I/II on the cell surface. SKG-II cells $\left(0.5 \times 10^{4}\right.$ cells $)$ were treated for $24 \mathrm{~h}$ with eEMP-I/II $(2 \mu \mathrm{g} / \mathrm{ml})$. After fixing the cells, eEMP-I/II was visualized using a mouse anti-(V5)IgG, which was then complexed with a tetramethylrhodamine isothiocyanate-conjugated rabbit anti-(mouse IgG) IgG (Sigma Chemical, St. Louis, MO). Cells were viewed with a fluorescence and differential-interference microscope furnished with a digital camera (Olympus Optical Co., Tokyo, Japan).

Co-culture of SKG-II cells and human uterine cervical fibroblasts. SKG-II cells and human uterine cervical fibroblasts (HUCF) were co-cultured at 1:1 cell ratios according to the method in our previous report (11). The co-cultured cells were washed once with PBS(-) and then treated for $24 \mathrm{~h}$ with or without eEMP-I/II (1 and $2 \mu \mathrm{g} / \mathrm{ml})$ in serum-free DMEM. In 
Table I. Synthetic peptides for two extracellular loop domains of EMMPRIN.

\begin{tabular}{lccl}
\hline Peptides & Domains & Position & \multicolumn{1}{c}{ Amino acid sequences } \\
\hline EM1 & I & $42-57$ & SLNDSATEVTGHRWLK \\
EM2 & I & $54-69$ & RWLKGGVVLKEDALPG \\
EM3 & I & $66-78$ & ALPGQKTEFKVDS \\
EM4 & I & $75-86$ & KVDSDDQWGEYS \\
EM5 & II & $127-141$ & KSESVPPVTDWAWYK \\
EM6 & II & $138-152$ & AWYKITDSEDKALMN \\
EM7 & II & $149-163$ & ALMNGSESRFFVSS \\
EM8 & II & $160-173$ & VSSSQGRSELHIEN \\
EM9 & II & $170-184$ & HIENLNMEADPGQYR \\
\hline
\end{tabular}

this series of experiments, HUCF were used at the 6th to 10th passage levels.

Western blot analysis. Purified eEMP-I/II and native EMMPRIN derived from SKG-II cells were confirmed by Western blot analysis using polyclonal antibodies against the N- (N19) and C-terminal regions (K20) of EMMPRIN (Santa Cruz Biotechnology, Santa Cruz, CA), and mouse anti-(V5)IgG (Sigma Chemical). Briefly, eEMP-I/II $(2 \mu \mathrm{g})$ and cell lysates (50 $\mu \mathrm{g}$ protein) prepared from SKG-II cells (13) were subjected to SDS-polyacrylamide gel electrophoresis (SDS-PAGE) using $15 \%$ acrylamide gel and then the proteins separated on the gels were electrotransferred onto the nitrocellulose membrane. The membranes were reacted with goat anti-(human EMMPRIN) IgGs (N19 and K20) or mouse anti-(V5)IgG. To evaluate the level of $\beta$-actin as an internal control in siRNA treatments, the harvested cell lysates (50 $\mu \mathrm{g}$ protein) were similarly subjected to Western blot analysis using rabbit anti-(human $\beta$-actin) IgG (Medical \& Biological Laboratories, Nagoya, Japan). For the analysis of proMMP-1 production, aliquots $(1.5 \mathrm{ml})$ of the harvested culture media were subjected to SDS-PAGE using $12.5 \%$ acrylamide gels, and after the electrotransfer, the membrane was reacted with sheep anti-(human proMMP-1) IgG (graciously provided by Professor H. Nagase, the Kennedy Institute of Rheumatology, The Imperial College London, London, UK) (11). Immunoreactive EMMPRIN, $\beta$-actin, and proMMP-1 were visualized with Amersham enhanced chemiluminescence-Western blotting detection reagents (GE Healthcare Bio-Sciences, Tokyo, Japan) according to the manufacturer's instructions. Relative amounts of proMMP-1 protein were quantified by densitometric scanning using an Image Analyzer LAS-1000 Plus (GE Healthcare), and the relative expression level was expressed as the mean value of the control as $100 \%$.

Statistical analysis. One-way analysis of variance (ANOVA) was used for statistical analysis. The Fisher test was applied when multiple comparisons were performed.

\section{Results}

EMMPRIN knockdown enhances the migration of human uterine cervical carcinoma SKG-II cells. To evaluate whether
A

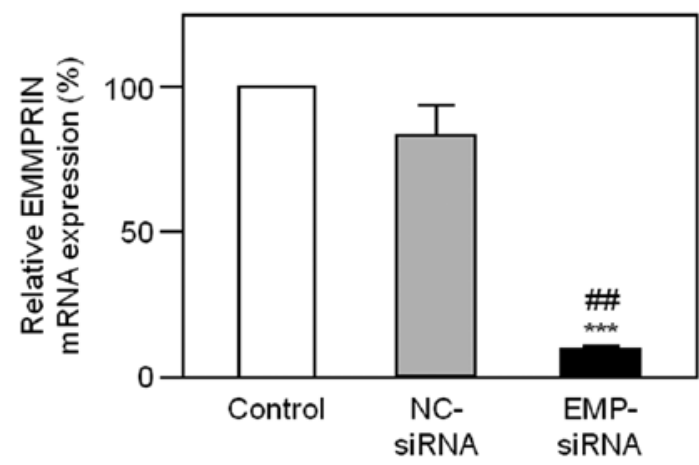

B

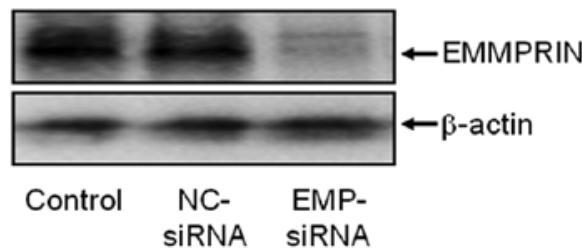

Figure 1. Silencing of EMMPRIN expression by siRNA-treatments in SKG-II cells. SKG-II cells were transfected for $24 \mathrm{~h}$ with or without negative control siRNA (NC-siRNA) (5 nM) and EMMPRIN siRNA (EMP-siRNA) (5 nM). (A) Total RNA isolated from the cells was subjected to real-time PCR for EMMPRIN mRNA as described in Materials and methods. ${ }^{* * *}$ Significantly different from untransfected cells (control) $(\mathrm{p}<0.001) .{ }^{\# /}$ Significantly different from negative control siRNA-treated cells $(\mathrm{p}<0.01)$. (B) Harvested cell lysates (50 $\mu \mathrm{g}$ protein) were subjected to Western blot analysis for EMMPRIN and $\beta$-actin as described in Materials and methods. Control, untransfected cells.

or not EMMPRIN participated in the regulation of tumor cell migration, we examined the effect of EMMPRIN siRNA on the migration of SKG-II cells, which constitutively expressed EMMPRIN on the cell surface (11). As shown in Fig. 1, the gene expression and production of EMMPRIN was suppressed in the EMMPRIN siRNA transfected SKG-II cells. In contrast, there were no changes in the levels of EMMPRIN mRNA and protein in the negative control siRNA transfected SKG-II cells. An in vitro scratch wound assay using EMMPRIN-knockdown SKG-II cells revealed the enhancement of the cell migration as compared with that of the negative control siRNA transfected cells (Fig. 2). Thus, these results suggest that the suppression of EMMPRIN expression facilitates cell migration in SKG-II cells.

eEMP-I/II augments SKG-II cell migration due to the functional interference of native EMMPRIN on the cell surface. The biological activities of EMMPRIN on the cell surface have been reported to be abolished by administration of an unglycosylated EMMPRIN mutant that inhibits the homophilic dimerization of native EMMPRIN on the cell surface (26). Therefore, to elucidate the negative regulation of cell migration by native EMMPRIN on the cell surface, we constructed an E. coli-expressed unglycosylated EMMPRIN mutant (termed eEMP-I/II) and examined the effect of eEMP-I/II on the migration of SKG-II cells. First, Western blot analysis revealed that the constructed eEMP-I/II was detectable at $\sim 28 \mathrm{kDa}$ by antibodies against the $\mathrm{N}$-terminal region of EMMPRIN (N19) and V5 tag, but not by an EMMPRIN antibody against its C-terminal region (K20) (Fig. 3A). In contrast, native EMMPRIN ( 53 kDa) prepared from SKG-II 
A
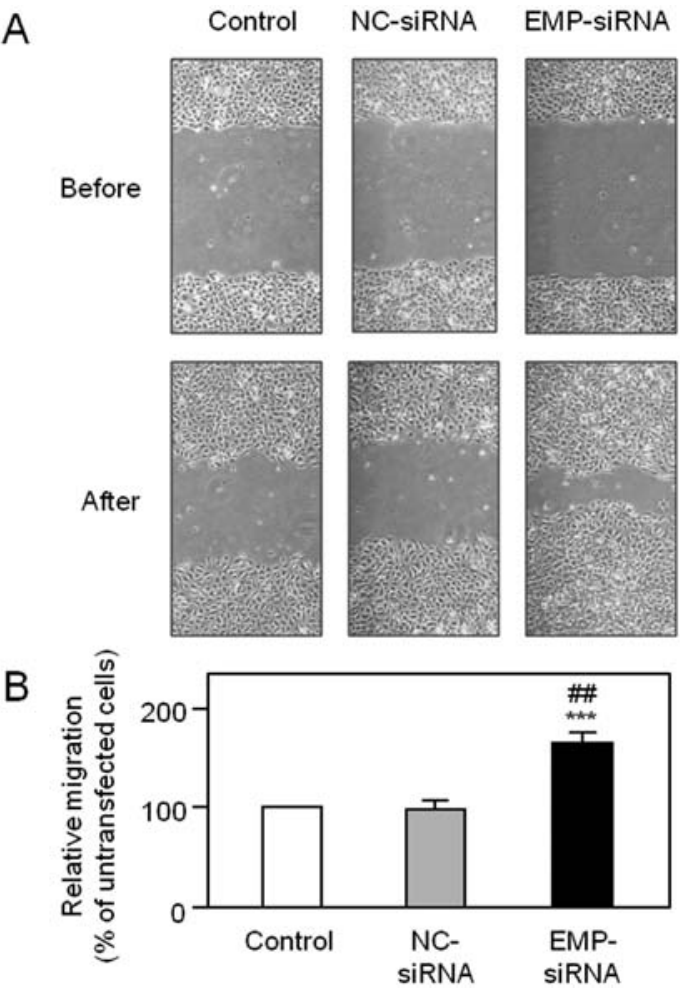

Figure 2. Augmentation of SKG-II cell migration by EMMPRIN siRNA. (A) Confluent SKG-II cells or siRNA-transfected SKG-II cells in 35-mm dishes were scratched with a pipet-tip (before) and then cultured for $14 \mathrm{~h}$ (after) as described in Materials and methods. Control, untransfected cells. NC-siRNA, negative control siRNA-transfected cells. EMP-siRNA, EMMPRIN siRNAtransfected cells. (B) Cell migration distance $(\mu \mathrm{m})$ before and after treatments was measured, and then relative cell migration was expressed by taking untransfected cells (control) as $100 \%$. Data are shown as mean \pm SD of six individual areas. ${ }^{* * *}$ Significantly different from control $(\mathrm{p}<0.001)$. cells were found to be detectable by antibodies against both the $\mathrm{N}$ - and C-terminal regions of EMMPRIN, but not by the V5 antibody. In addition, immuno-cytochemical analysis using the V5 antibody showed that eEMP-I/II was detectable on the cell surface of the SKG-II cells (arrows in Fig. 3B). The administration of eEMP-I/II was found to facilitate SKG-II cell migration in a dose-dependent manner (1.6-fold at $2 \mu \mathrm{g} / \mathrm{ml})$ (Fig. 3C and D). Moreover, to elucidate whether eEMP-I/II functionally interfered with the actions of native EMMPRIN on the cell surface, we examined the effect of eEMP-I/II on the EMMPRIN-mediated augmentation of proMMP-1 production in a co-culture of SKG-II cells and HUCF (11). As shown in Fig. 4, eEMP-I/II was found to dose-dependently inhibit the native EMMPRIN-mediated augmentation of proMMP-1 production in the co-culture of SKG-II cells and HUCF. Taken together, these results suggest that the extracellularly administered eEMP-I/II functionally interfered with native EMMPRIN on the cell surface, which in turn enhances SKG-II cell migration.

Identification of a functional site of EMMPRIN that negatively regulates the cell migration of SKG-II cells. Our previous study of a systematic peptide screening method using nine EMMPRIN peptides (EM1-9) (positions: 42-184 amino acids in the extracellular regions, not including the hinge region between 87-126 amino acids) revealed that ${ }^{42}$ SLNDSATEVTGHRWLK ${ }^{57}$ (EM1) in the loop I domain of native EMMPRIN was involved in the augmentation of MMP production (11). To identify the crucial site(s) of native EMMPRIN that negatively regulates cell migration, an in vitro wound assay was performed in the presence of nine EMMPRIN peptides (EM1-9) (Table I). Among these peptides, EM9, ${ }^{170} \mathrm{HIENLNMEADPGQYR}{ }^{184}$, in the loop

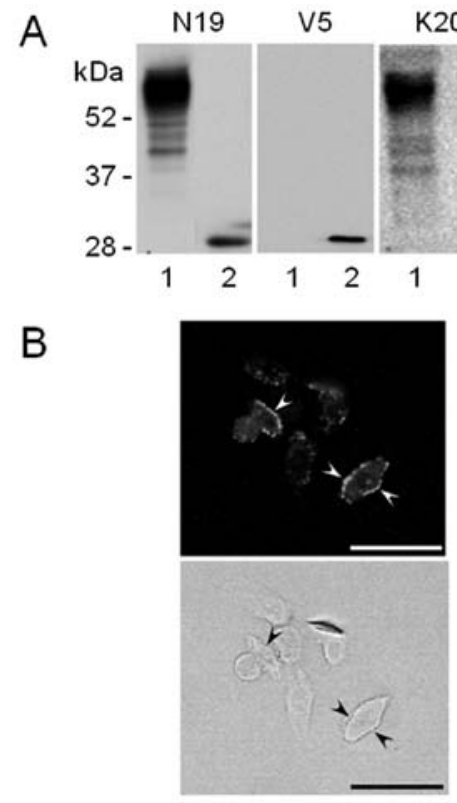

\section{C}

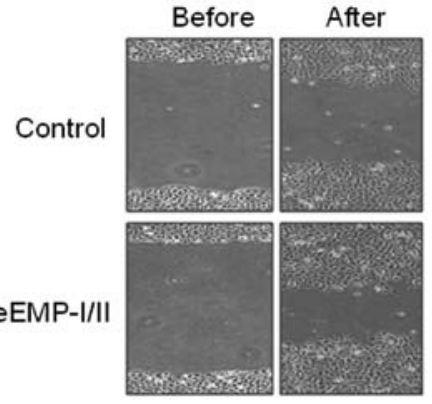

D

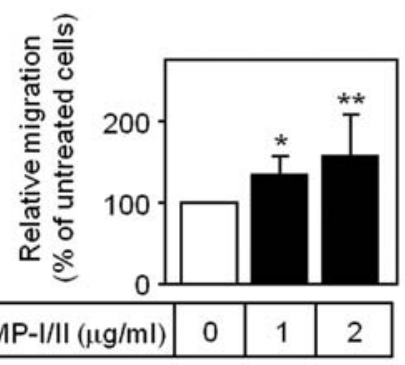

Figure 3. Enhancement of SKG-II cell migration by eEMP-I/II. (A) Western blot analysis revealed that native EMMPRIN derived from SKG-II cells (arrow) and eEMP-I/II (arrowhead) were detectable by an antibody against the N-terminal region of EMMPRIN (N19), whereas only native EMMPRIN was detectable by an antibody against the C-terminal region of EMMPRIN (K20). eEMP-I/II, but not native EMMPRIN, was also detectable by V5-tag antibody (V5). Lane 1, cell lysate (50 $\mu \mathrm{g}$ protein) prepared from SKG-II cells and lane 2, eEMP-I/II $(2 \mu \mathrm{g})$. (B) When SKG-II cells were treated for $24 \mathrm{~h}$ with eEMP-I/II $(2 \mu \mathrm{g} / \mathrm{ml})$, eEMP-I II was immunologically detectable on the cell surface of SKG-II cells (arrows) using a mouse anti-(V5)IgG (upper panel). Lower panel, light microscopy of the same cells as in the upper panel. Bars, $100 \mu \mathrm{m}$. (C) Scratched SKG-II cells as shown in Fig. 2 were treated for $14 \mathrm{~h}$ with or without eEMP-I/II (2 $\mu \mathrm{g} / \mathrm{ml})$. The behavior of SKG-II cell migration before and after treatments of eEMP-I/II are shown. (D) Cell migration distance ( $\mu \mathrm{m})$ before and after treatments as shown in (C) was measured, and then relative cell migration was expressed by taking untreated cells as $100 \%$. Data are shown as mean \pm SD of six individual areas. ${ }^{* * * *}$ Significantly different from the untreated cells ( $\mathrm{p}<0.05$ and 0.001 , respectively). 


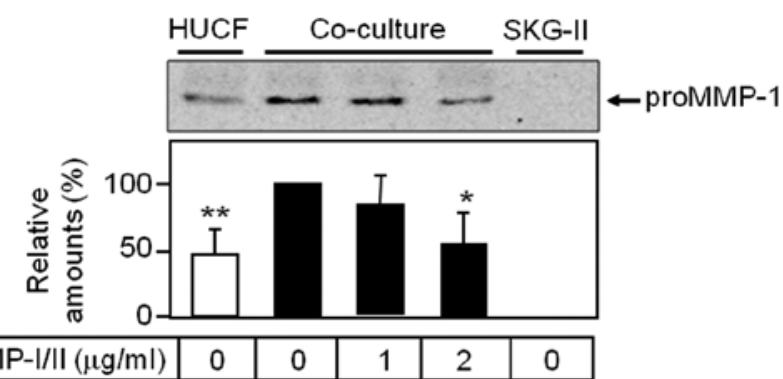

Figure 4. Inhibition of EMMPRIN-induced proMMP-1 production by eEMP-I/II in a co-culture of SKG-II cells and human uterine cervical fibroblasts. Human uterine cervical fibroblasts (HUCF) were co-cultured with SKG-II cells in a 1:1 cell ratio, and then the co-cultured cells were treated for $24 \mathrm{~h}$ with or without eEMP-I/II ( 1 and $2 \mu \mathrm{g} / \mathrm{ml})$. The harvested culture media were subjected to Western blot analysis for proMMP-1. The relative amounts of proMMP-1 were quantified by densitometric scanning and expressed by taking untreated cells in the co-culture as $100 \%$. Data are shown as mean \pm SD of three independent experiments. ${ }^{* * *}$ Significantly different from the untreated cells in the co-culture ( $<<0.05$ and 0.01 , respectively).

II domain of EMMPRIN was found to significantly augment SKG-II cell migration (Fig. 5A and B). However, there was no or a negligible effect in the cells treated with other peptides (Fig. 5A). In addition, the enhancement of cell migration by the EM9 peptide was found to be dose-dependent (Fig. 5C). Furthermore, SKG-II cell migration was dose-dependently facilitated by administration of an antibody against EM9, but not one against EM1 (Fig. 6). Therefore, these results suggest that EM9, ${ }^{170} \mathrm{HIENLNMEADPGQYR}{ }^{184}$, in the second loop domain of native EMMPRIN, was involved in the limitation of cell migration in SKG-II cells.

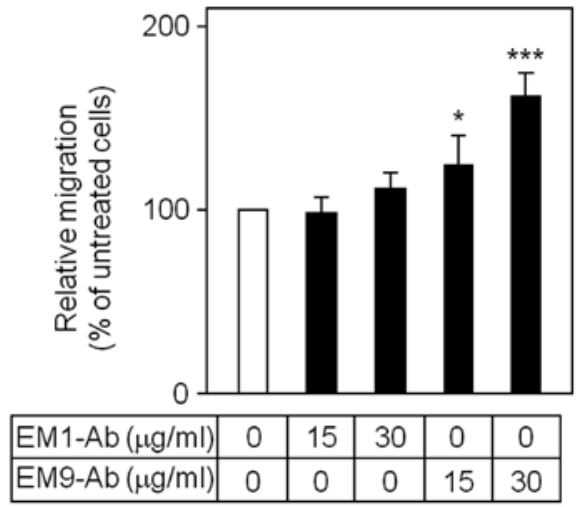

Figure 6. Effects of EMMPRIN antibodies on SKG-II cell migration. Scratched SKG-II cells as shown in Fig. 2 were treated for $14 \mathrm{~h}$ with or without antibodies against EM1 (EM1-Ab) and EM9 (EM9-Ab) (15 and $30 \mu \mathrm{g} / \mathrm{ml})$, and then relative cell migration was expressed by taking untreated cells as $100 \%$. Data are indicated as mean $\pm \mathrm{SD}$ of three individual areas. ${ }^{* * *}$ Significantly different from the untreated cells $(\mathrm{p}<0.05$ and 0.001 , respectively).

\section{Discussion}

The enhancement of tumor cell migration has been associated with the progression of tumor invasion and metastasis $(17,18)$. Regarding the association of EMMPRIN with cell migration, CD147/EMMPRIN has been reported to be involved in T-lymphocyte chemotaxis leading to inflammatory reactions (27). In addition, Hanata et al (19) have reported that soluble full-length EMMPRIN from human laryngeal epidermoid carcinoma HEp-2 cells is indirectly associated with the augmentation of tumor-cell migration in a separate co-culture model with fibroblasts. A recent study by Dang et al (28) has shown that

A

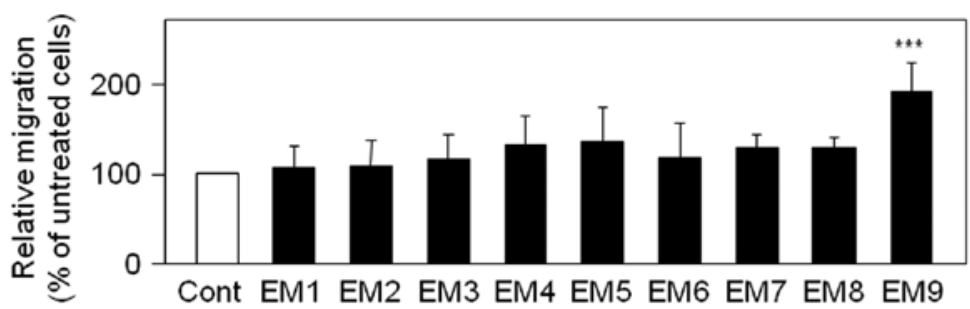

B

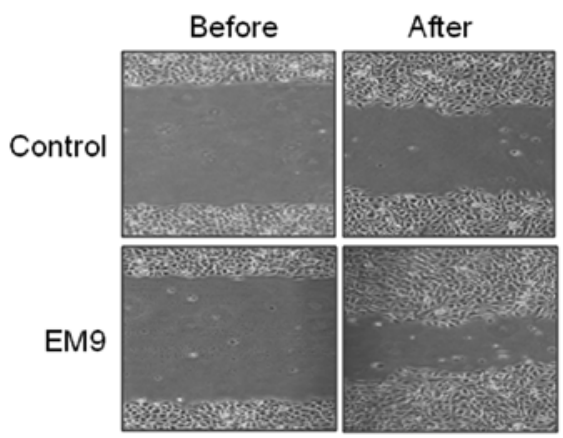

C

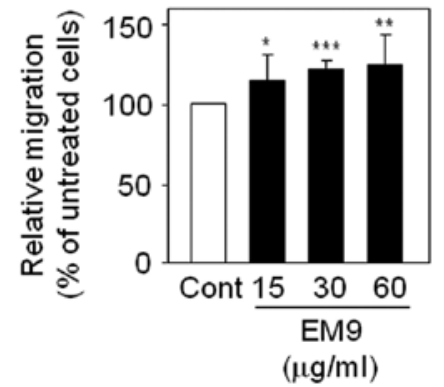

Figure 5. Effects of synthetic EMMPRIN peptides on SKG-II cell migration. (A) Scratched SKG-II cells as shown in Fig. 2 were treated for $14 \mathrm{~h}$ with or without nine synthetic EMMPRIN peptides (EM1-9) $(60 \mu \mathrm{g} / \mathrm{ml})$, and then relative cell migration was expressed by taking untreated cells as $100 \%$. (B) The behavior of SKG-II cell migration before and after treatments of EM9 peptide $(60 \mu \mathrm{g} / \mathrm{ml})$. (C) Scratched SKG-II cells were similarly treated for $14 \mathrm{~h}$ with or without EM9 peptide $(15-60 \mu \mathrm{g} / \mathrm{ml})$, and then relative cell migration was expressed by taking untreated cells as $100 \%$. Data are shown as mean \pm SD of six individual areas. ${ }^{*, * * * * * *}$ Significantly different from the untreated cells (cont) $(\mathrm{p}<0.05,0.01$, and 0.001 , respectively). 
the overexpression of EMMPRIN facilitates and EMMPRIN siRNA suppresses the migration of squamous cell carcinoma cells. In the present study, EMMPRIN siRNA suppressed the gene expression and production of EMMPRIN in human uterine cervical carcinoma SKG-II cells, which constitutively express EMMPRIN on the cell surface (11). Furthermore, the migration activity of SKG-II cells was enhanced by adding EMMPRIN siRNA. Therefore, native EMMPRIN on the cell surface is likely to limit the cell migration of SKG-II cells. Moreover, the differential regulation of cell migration by EMMPRIN may be due to tumor cell species and/or experimental conditions.

It has been reported that native EMMPRIN-induced proMMP-1 production in human dermal fibroblasts is abolished by administration of purified deglycosylated-EMMPRIN that inhibits the homophilic dimerization of EMMPRIN on the cell surface (26). In addition, EMMPRIN-dependent cell adhesion has been reported to be inhibited by a soluble EMMPRIN fusion protein that consists of two extracellular loop domains and mouse IgG2a Fc (26). Thus, this report allows us to hypothesize that the E. coli-expressed EMMPRIN mutant with the extracellular domains, eEMP-I/II, is a useful competitor for the evaluation of native EMMPRIN functions on the cell surface. In the present study, the administration of eEMP-I/II was found to cause the enhancement of SKG-II cell migration. Furthermore, like in the case of a previous report (26), we demonstrated that eEMP-I/II suppresses the native EMMPRIN-induced proMMP-1 production in the co-culture of SKG-II cells and HUCF. Taken together with our finding that eEMP-I/II is detectable on the cell surface of SKG-II cells, therefore, it can be suggested that eEMP-I/II exhibits the augmentation of SKG-II cell migration by possible mechanisms that interfere with the homophilic and/or heterophilic dimerization of native EMMPRIN on the cell surface.

Two extracellular loop domains of EMMPRIN have been reported to exhibit various biological functions. For instance, the loop I domain of EMMPRIN has been associated with MMP inducible activity and homophilic dimerization (1). A recent study of ours has shown that the amino acid sequence, ${ }^{42}$ SLNDSATEVTGHRWLK $^{57}$ (EM1) in the loop I domain of EMMPRIN participates in the augmentation of proMMP-1 production in HUCF co-cultured with SKG-II cells (11). In the present study, neither the EM1 peptide nor another three peptides coding the loop I domain of EMMPRIN (EM2-4) influenced the SKG-II cell migration. Thus, the loop I domain of EMMPRIN is unlikely to contribute to the regulation of cell migration in SKG-II cells. On the other hand, the loop II domain of EMMPRIN has been reported to form a complex with caveolin-1 and cyclophilin $(14,16)$. In the present study, we found that the synthetic EM9 peptide of EMMPRIN, ${ }^{170} \mathrm{HIENLNMEADPGQYR}{ }^{184}$, which exists close to the $\mathrm{C}$-terminal end of the loop II domain, enhances the migration activity of SKG-II cells. In addition, the administration of an EM9 antibody, but not an EM1 antibody, augmented SKG-II cell migration. Therefore, these results suggest for the first time that ${ }^{170} \mathrm{HIENLNMEADPGQYR}{ }^{184}$ in the loop II domain of EMMPRIN is a pivotal site for the limitation of cell migration in SKG-II cells.

Although EMMPRIN-binding molecules on the cell surface have been reported (e.g., integrins and EMMPRIN itself) $(1,2)$, to the best of our knowledge, there have been no reports on cell-surface candidate(s) recognizing the EM9 sequence in EMMPRIN. A recent study by Hanoulle et al (16) reported that the cis/trans isomerization of $\mathrm{Pro}^{180}$ in CD147 by cyclophilin B is required for the CD147-associated chemotactic activity in T lymphocytes. In addition to our confirmation that $\mathrm{Pro}^{180}$ is contained in the EM9 sequence, we have demonstrated that cyclophilin B is constitutively expressed but does not interact with EMMPRIN in SKG-II cells (data not shown). Thus, further experiments are needed to identify the membraneassociated molecule(s) that interacts with the EM9 sequence of EMMPRIN.

How does the limitation of tumor cell migration by native EMMPRIN play a role in the invasion and metastasis of cervical cancers? In this regard, we hypothesize that the limitation of tumor cell migration by EMMPRIN leads to the activation of cell-cell interaction between the tumor and adjacent stroma cells, which progresses the degradation of peritumoral extracellular matrix (ECM) due to the augmentation of MMP production. Thereafter, the alteration of peritumoral ECM may trigger the dissociation of cell-ECM interaction and the heterophilic dimerization of EMMPRIN through adhesion molecules such as integrins, which in turn facilitate the dissemination, migration, and invasion of tumor cells in vivo $(29,30)$. This hypothesis can be supported by previous reports that: i) the integrin-dependent signal stimulates the malignant transformation and invasive phenotype in human cervical carcinoma (31,32), and ii) EMMPRIN binds to integrin $\alpha 3 \beta 1$ and $\alpha 6 \beta 1$ in some tumor cell lines (33-35). Although we have demonstrated that integrin $\alpha 3 \beta 1$ is detectable in the cell surface of SKG-II cells (data not shown), further experiments will be needed to clarify this hypothesis.

In conclusion, we demonstrated that SKG-II cell migration was augmented by administering EMMPRIN siRNA, eEMP-I/II, synthetic EMMPRIN peptides, and a specific antibody against the EM9 sequence in the second loop domain of EMMPRIN, which transcriptionally and competitively diminished EMMPRIN functions on the cell surface of human cervical carcinoma SKG-II cells. Therefore, these results provide novel evidence that EMMPRIN on the cell surface limits the cell migration of human uterine cervical carcinoma cells through ${ }^{170}$ HIENLNMEADPGQYR ${ }^{184}$ in the loop II domain. Finally, these results should provide an increased understanding of the functions of EMMPRIN in malignant cervical carcinoma cells, and could contribute to the development of clinical strategies for cervical cancer therapy.

\section{Acknowledgements}

This work was supported in part by a grant to private universities provided by the Promotion and Mutual Aid Corporation for Private Schools of Japan, and by a Grant-in-Aid for Scientific Research (C) (\#22590506). We wish to thank Prof. H. Nagase of the Kennedy Institute of Rheumatology, The Imperial College London, London, UK for graciously providing the sheep anti-(human proMMP-1)IgG, and Mr. S. Konno for his technical assistance.

\section{References}

1. Nabeshima K, Iwasaki H, Koga K, et al: Emmprin (basigin/CD147): matrix metalloproteinase modulator and multifunctional cell recognition molecule that plays a critical role in cancer progression. Pathol Int 56: 359-367, 2006 
2. Iacono KT, Brown AL, Greene MI, et al: CD147 immunoglobulin superfamily receptor function and role in pathology. Exp Mol Pathol 83: 283-295, 2007.

3. Koch C, Staffler G, Hüttinger R, et al: T cell activation-associated epitopes of CD147 in regulation of the T cell response, and their definition by antibody affinity and antigen density. Int Immunol 11: 777-786, 1999.

4. Liang L, Major T and Bocan T: Characterization of the promoter of human extracellular matrix metalloproteinase inducer (EMMPRIN). Gene 282: 75-86, 2002.

5. Noguchi Y, Sato T, Hirata M, et al: Identification and characterization of extracellular matrix metalloproteinase inducer in human endometrium during the menstrual cycle in vivo and in vitro. J Clin Endocrinol Metab 88: 6063-6072, 2003.

6. Muraoka K, Nabeshima K, Murayama T, et al: Enhanced expression of a tumor-cell-derived collagenase-stimulatory factor in urothelial carcinoma: its usefulness as a tumor marker for bladder cancers. Int J Cancer 55: 19-26, 1993.

7. Polette M, Gilles C, Marchand V, et al: Tumor collagenase stimulatory factor (TCSF) expression and localization in human lung and breast cancers. J Histochem Cytochem 45: 703-709, 1997.

8. Bordador LC, Li X, Toole BP, et al: Expression of emmprin by oral squamous cell carcinoma. Int J Cancer 85: 347-352, 2000.

9. Sameshima T, Nabeshima K, Toole BP, et al: Expression of emmprin (CD147), a cell surface inducer of matrix metalloproteinases, in normal human brain and gliomas. Int J Cancer 88: 21-27, 2000.

10. Klein CA, Seidl S, Petat-Dutter K, et al: Combined transcriptome and genome analysis of single micrometastatic cells. Nat Biotechnol 20: 387-392, 2002 .

11. Sato T, Ota T, Watanabe M, et al: Identification of an active site of EMMPRIN for the augmentation of matrix metalloproteinase-1 and -3 expression in a co-culture of human uterine cervical carcinoma cells and fibroblasts. Gynecol Oncol 114: 337-342, 2009.

12. Koga K, Nabeshima K, Aoki M, et al: Emmprin in epithelioid sarcoma: expression in tumor cell membrane and stimulation of MMP-2 production in tumor-associated fibroblasts. Int J Cancer 120: 761-768, 2007.

13. Tang W, Chang SB and Hemler ME: Links between CD147 function, glycosylation, and caveolin-1. Mol Biol Cell 15: 4043-4050, 2004.

14. Tang W and Hemler ME: Caveolin-1 regulates matrix metalloproteinases-1 induction and CD147/EMMPRIN cell surface clustering. J Biol Chem 279: 11112-11118, 2004

15. Schwab W, Harada H, Goetz W, et al: Immunocytochemical and biochemical detection of EMMPRIN in the rat tooth germ: differentiation-dependent co-expression with MMPs and co-localization with caveolin-1 in membrane rafts of dental epithelial cells Histochem Cell Biol 128: 195-203, 2007.

16. Hanoulle X, Melchior A, Sibille N, et al: Structural and functional characterization of the interaction between cyclophilin B and a heparin-derived oligosaccharide. J Biol Chem 282: 34148-34158, 2007.

17. Hood JD and Cheresh DA: Role of integrins in cell invasion and migration. Nat Rev Cancer 2: 91-100, 2002.

18. Gassmann P, Enns A and Haier J: Role of tumor cell adhesion and migration in organ-specific metastasis formation. Onkologie 27: $577-582,2004$
19. Hanata K, Yamaguchi N, Yoshikawa K, et al: Soluble EMMPRIN (extra-cellular matrix metalloproteinase inducer) stimulates the migration of HEp-2 human laryngeal carcinoma cells, accompanied by increased MMP-2 production in fibroblasts. Arch Histol Cytol 70: 267-277, 2007.

20. Chen X, Su J, Chang J, et al: Inhibition of CD147 gene expression via RNA interference reduces tumor cell proliferation, activation, adhesion, and migration activity in the human Jurkat T-lymphoma cell line. Cancer Invest 26: 689-697, 2008.

21. Du ZM, Hu CF, Shao Q, et al: Upregulation of caveolin-1 and CD147 expression in nasopharyngeal carcinoma enhanced tumor cell migration and correlated with poor prognosis of the patients. Int J Cancer 125: 1832-1841, 2009.

22. Zhao P, Zhang W, Tang J, et al: Annexin II promotes invasion and migration of human hepatocellular carcinoma cells in vitro via its interaction with HAb18G/CD147. Cancer Sci 101: 387-395, 2010.

23. Parkin DM, Bray F, Ferlay J, et al: Global cancer statistics 2002. CA Cancer J Clin 55: 74-108, 2005.

24. Sier CF, Zuidwijk K, Zijlmans HJ, et al: EMMPRIN-induced MMP-2 activation cascade in human cervical squamous cell carcinoma. Int J Cancer 118: 2991-2998, 2006.

25. Ju XZ, Yang JM, Zhou XZ, et al: EMMPRIN expression as a prognostic factor in radiotherapy of cervical cancer. Clin Cancer Res 14: 494-501, 2008.

26. Sun $J$ and Hemler ME: Regulation of MMP-1 and MMP-2 production through CD147/extracellular matrix metalloproteinase inducer interactions. Cancer Res 61: 2276-2281, 2001.

27. Damsker JM, Bukrinsky MI, Constant SL: Preferential chemotaxis of activated human $\mathrm{CD} 4^{+} \mathrm{T}$ cells by extracellular cyclophilin A. J Leukoc Biol 82: 613-618, 2007.

28. Dang D, Atakilit A and Ramos DM: EMMPRIN modulates migration and deposition of $\mathrm{TN}-\mathrm{C}$ in oral squamous carcinoma. Anticancer Res 28: 2049-2054, 2008.

29. Moh MC and Shen S: The roles of cell adhesion molecules in tumor suppression and cell migration: a new paradox. Cell Adh Migr 3: 334-336, 2009.

30. Box C, Rogers SJ, Mendiola M, et al: Tumour-microenvironmental interactions: paths to progression and targets for treatment. Semin Cancer Biol 20: 128-138, 2010.

31. Oktay MH, Oktay K, Hamele-Bena D, et al: Focal adhesion kinase as a marker of malignant phenotype in breast and cervical carcinomas. Hum Pathol 34: 240-245, 2003.

32. Maity G, Sen T and Chatterjee A: Laminin induces matrix metalloproteinase-9 expression and activation in human cervical cancer cell line (SiHa). J Cancer Res Clin Oncol 137: 347-357, 2011.

33. Berditchevski F, Chang S, Bodorova J, et al: Generation of monoclonal antibodies to integrin-associated proteins. Evidence that $\alpha 3 \beta 1$ complexes with EMMPRIN/basigin/OX47/M6. J Biol Chem 272: 29174-29180, 1997.

34. Tang J, Wu YM, Zhao P, et al: Overexpression of HAb18G/CD147 promotes invasion and metastasis via $\alpha 3 \beta 1$ integrin mediated FAK-paxillin and FAK-PI3K-Ca ${ }^{2+}$ pathways. Cell Mol Life Sci 65: 2933-2942, 2008

35. Dai JY, Dou KF, Wang CH, et al: The interaction of HAb18G/ CD147 with integrin $\alpha 6 \beta 1$ and its implications for the invasion potential of human hepatoma cells. BMC Cancer 9: 337, 2009. 